\title{
Predicting the temporal activity patterns of new venues
}

\author{
Krittika D'Silva ${ }^{1 *}$ (D), Anastasios Noulas², Mirco Musolesi ${ }^{3,4}$, Cecilia Mascolo ${ }^{1,4}$ and Max Sklar ${ }^{5}$
}

\author{
${ }^{*}$ Correspondence: \\ krittika.dsilva@cl.cam.ac.uk \\ 'Department of Computer Science, \\ University of Cambridge, \\ Cambridge, UK \\ Full list of author information is \\ available at the end of the article
}

\begin{abstract}
Estimating revenue and business demand of a newly opened venue is paramount as these early stages often involve critical decisions such as first rounds of staffing and resource allocation. Traditionally, this estimation has been performed through coarse-grained measures such as observing numbers in local venues or venues at similar places (e.g., coffee shops around another station in the same city). The advent of crowdsourced data from devices and services carried by individuals on a daily basis has opened up the possibility of performing better predictions of temporal visitation patterns for locations and venues. In this paper, using mobility data from Foursquare, a location-centric platform, we treat venue categories as proxies for urban activities and analyze how they become popular over time. The main contribution of this work is a prediction framework able to use characteristic temporal signatures of places together with $k$-nearest neighbor metrics capturing similarities among urban regions, to forecast weekly popularity dynamics of a new venue establishment in a city neighborhood. We further show how we are able to forecast the popularity of the new venue after one month following its opening by using locality and temporal similarity as features. For the evaluation of our approach we focus on London. We show that temporally similar areas of the city can be successfully used as inputs of predictions of the visit patterns of new venues, with an improvement of $41 \%$ compared to a random selection of wards as a training set for the prediction task. We apply these concepts of temporally similar areas and locality to the real-time predictions related to new venues and show that these features can effectively be used to predict the future trends of a venue. Our findings have the potential to impact the design of location-based technologies and decisions made by new business owners.
\end{abstract}

Keywords: Human mobility prediction; Urban traffic; Spatio-temporal patterns; Urban computing

\section{Introduction}

Cities are complex systems that constantly change over time. From city dwellers that commute to work on a weekday morning to visitors who arrive in town for business or leisure, the urban landscape is transforming at a fast pace. The way in which city neighborhoods become popular over time has been a fundamental area of study in traditional urban studies literature as it is critical to city governance $[1,2]$. The rise of mobile technologies and collective sensing in the last decade has contributed to the generation of large datasets that describe activity dynamics in cities and has created new opportunities for research in the

(c) The Author(s) 2018. This article is distributed under the terms of the Creative Commons Attribution 4.0 International License (http://creativecommons.org/licenses/by/4.0/), which permits unrestricted use, distribution, and reproduction in any medium, provided you give appropriate credit to the original author(s) and the source, provide a link to the Creative Commons license, and indicate if changes were made. 
area; for example, a number of works have proposed the use of cellular data to understand collective mobility dynamics and inform planning decisions [3-7]. Beyond cellular data, the increasing popularity of services like Twitter and Foursquare has yielded new inputs for capturing the heartbeat of a city [8-10]. Further, trends in credit card transactions have also been used to study human patterns across space and time [11].

On the venue level, temporal dynamics and the spatial configuration of urban activities has helped decide where to geographically place new retail facilities $[12,13]$ as well as to power mobile applications such as local search [14] by exploiting place temporal dynamics. Nevertheless, little work has looked at predicting what happens after a new venue opens in a city neighborhood; will it become popular? and moreover, at which times of the week should the owner of a retail facility expect high volumes of customer traffic? This information is important during the early stages of a new business when staffing levels must be decided, supplies bought, and opening times established.

In this paper, starting from the premise that mobility in a city is driven by local urban activities, we provide an analytical framework that captures the popularity dynamics of urban neighborhoods. We then exploit these temporal patterns across areas to predict the popularity dynamics of newly established venues. The primary data input we use for our study is a longitudinal dataset from location-based service Foursquare ${ }^{\mathrm{a}}$ describing mobility in terms of user check-ins at public venues in the city of London. Our approach can be summarized as follows:

- Temporal characterization of urban activities across regions: First, we show how the temporal profile of an area in terms of the number of mobile users that visit over the course of a week varies significantly from neighborhood to neighborhood. These temporal profiles are shaped by the daily and weekly circadian rhythms of moving populations as well as their choice of specific urban activities at key times. Further, we demonstrate how the popularity dynamics of venue categories give rise to the temporal patterns of the urban areas that contain them, highlighting how urban activities and population levels at a neighborhood are inherently interconnected temporal processes.

- Predicting the popularity dynamics of newly established venues: Next, we harness the temporal popularity patterns of venue categories to predict the weekly temporal profile of a newly established venue. Our approach is based on two key principles. First, we exploit the fact that despite their differences, neighborhoods in a city can exhibit a high degree of temporal synchronicity, even if they are geographically located far from one another. For example, the city of London includes neighborhoods such as SoHo and Camden Town, both of which attract a young late-night crowd. These shared traits could mean these neighborhoods are likely to become popular at similar times. Second, we make use of the principle of locality that suggests that the temporal profile of venues in the same neighborhood are highly correlated as individual movements in a city tend to be constrained by distance. We combine the properties of synchronicity and locality in a novel $k$-nearest neighbor model to determine similarities between neighborhoods in cities and use this understanding to predict the characteristic demand curve of a new venue. We use classic Gaussian Processes along with a $k$-nearest neighbor approach to build a model which accurately predicts a new venue's popularity temporal profile. Our results perform significantly better than our random baseline, decreasing the normalized root mean square error by $41 \%$. 
- Real time demand prediction of new venues: Finally, given a new venue, our goal becomes to predict how the demand changes as that venue matures over time. With each progressive month of growth for a new venue, it is to be expected that some venues flourish and grow in popularity over time while others may be less successful and see their demand decline over subsequent months. Although sparsity can be an issue when working at a fine spatial granularity, making the formulation of a regression problem challenging, we show that it is possible to improve the prediction of expected levels of visits for the next time step by using an approach based on neighborhood synchronicity and locality. We train a Gaussian Process model on the month to month trends of check-ins to other venues and wards and use these as inputs to forecast the popularity of a new venue. In doing so, we incorporate recent changes in urban mobility, which could arise for instance due to the presence of new events nearby or other anomalies such as transport disruptions.

Our work enables a fine-grained dynamic estimation of activity for new venues. Obtaining analytics in this context can help can business owners predict demand in dynamics for their business and therefore plan better the provision of services to their customers.

The remainder of the paper is organized as follows. In Sect. 2, we motivate our work and introduce the related work in the area. Section 3 gives an overview of our approach and Sect. 4 introduces a formalization of our framework. Section 5 reports on our temporal analysis of venues and neighborhoods in London. In Sect. 6, we describe a method for predicting the characteristic temporal profiles of a set of new venues using a batch-learning approach, whereas in Sect. 7 we present an analysis of the real-time extension of this approach. We conclude the paper with Sect. 8 discussing our results and Sect. 9 highlighting possible future work.

\section{Related work}

The ubiquity of GPS sensors on mobile devices as well as the introduction of the mobile web have been game changers with respect to the scales and types of data available about human mobility. The rise of services such as Foursquare and more generally applications that rely on geo-tagging technologies (e.g. Twitter, Instagram, Flickr) combined with the accessibility to the corresponding APIs have offered novel views to collective mobility activity in cities $[15,16]$. This has led to more granular representations of urban activities across space and time [8-10], and has been used to characterize cities in terms of their urban growth patterns [17] and cultural boundaries in terms of their culinary patterns [18]. Additionally, such location-based technologies have significantly improved the quality of experience for mobile users as they navigate the city. Foursquare's data science team exploited the weekly temporal visitation patterns of venues to power its local search engine [14]. Google Maps recently incorporated the feature popular times that appears when search results about places are shown to users [19], while Facebook launched its in-house place search service [20]. The commercialization potential of such services has naturally expanded beyond the realm of location-based technologies and has contributed to coining the term location intelligence when referring to business intelligence relying on geospatial data. In this direction, a number of works have appeared on retail optimization in cities, including the identification of the best location to open a new shop $[12,13]$ or the ranking of areas according to their real estate value [21].

Before datasets from location-based services became available, those collected from cellular networks paved the way for understanding the collective dynamics of urban activities. 
Ratti et al. in [5] present one of the first works that demonstrates how urban landscapes transform in real time as populations move around the city. Beyond dynamic visualizations, the authors in $[3,4]$ characterize in statistical terms urban activity variations and provide interpretation on the observed patterns in terms of the underlying urban activities, such as transport and residential land uses, which drive population volumes regionally. Becker et al. in [6] use cellular data to characterize mobility trends across different metropolitan areas, while the authors in [7] propose using cellular data as an alternative to travel surveys so that more accurate spatio-temporal representations of mobility flows are obtained. Urban transport data has often been another source for capturing city dynamics $[22,23]$.

While in many of the works mentioned above, information on the temporal visitation patterns of users to locations has been used as input, none has looked at predicting the temporal signatures of visits to venues per se. This is where the primary novelty of the present paper lies. Considering the temporal patterns of user visits at newly established venues as our main prediction task we are hoping to offer new insights on location-based analytics for business owners that would empower them to make more informed choices on staffing, provision of goods, resources in the early days of their new business. We also envision the use of similar approaches to inform a number of tasks that are applicable in the urban domain. These may include the spatial deployment of taxi fleets and pooling services $[24,25]$ or the allocation of police or ambulance resources [26, 27].

\section{Our approach at a glance}

In this section, we describe how our approach to the prediction of visitation patterns to new venues harnesses temporal similarities of urban neighborhoods. Our analysis revolves around the concept of a characteristic weekly temporal profile, a time series representing typical changes in demand of a given entity over the course of a week. We explore characteristic profiles of venues, which fluctuate based on user mobility patterns, and neighborhoods that are composed of venue profiles.

We begin with the premise that venue categories have different characteristic weekly temporal profiles. These profiles represent variations in demand based on a user's propensity to visit that category at a given hour of the week. For example, the category of Travel \& Transport is likely to correlate with changes in rush hour traffic while Food could instead be dependent on typical meal times. Different neighborhoods in a city have characteristic weekly temporal profiles which are made up of contributions from different categories. We posit that neighborhoods which have similar temporal profiles or similar contributions of venues to their temporal profile could be predictors for each other. We apply this idea towards the analysis of new venues: given a new venue in a given neighborhood in a city, can we use the demand profile of venues in temporally similar wards as predictors for our new venue of interest? For new venues opening up in a city, often no prior information is known about the expected popularity or demand dynamics. The ability to approximate and better understand these metrics can be crucial for the success of a new business owner.

This characteristic temporal curve provides a static representation of the typical changes in demand of a venue over the course of a week. We build upon our analysis of new venues by dynamically predicting how the demand of a venue will change. Starting from one week after a venue has opened, we show that we can use data from venues with similar characteristic curves to more accurately predict the changes in demand at the next time step. 


\section{Notation and definitions}

\subsection{Dataset}

Online Location-based Social Networks (LBSNs) have recently experienced a surge in popularity, attracting millions of users around the world. The widespread adoption of these services in addition to location-sensing mobile devices has created a wealth of data about the mobility of humans in cities. Foursquare, a popular location-centric media platform, enables users to check into different locations and share that information with their friend group. As of August 2015, Foursquare had more than 50 million active users and more than 10 billion check-ins [28].

For our work we use a longitudinal dataset describing urban mobility and activity patterns in Greater London that spans three years and millions of check-ins. For each venue, we have the following information: geographic coordinates, specific and general category, creation date, total number of check-ins, and number of unique visitors. The specific and general categories fall within Foursquare's API of hierarchical categories. A full list of the categories can be found by querying the Foursquare API ${ }^{\mathrm{b}}$. General categories are overarching groups to one of which each specific category is assigned. Examples of general categories could include Food or Travel E Transport while examples of specific categories could be Chinese Restaurants or Italian Restaurants, which both aptly fall under the category of Food. In addition to data about venues, the dataset also contains transitions within London. A transition is defined as a pair of check-ins by an anonymous user to two different venues within the span of three hours. A transition is identified by a start time, end time, source venue, and destination venue. Our dataset includes 18,018 venues and 4,000,040 transitions for Greater London. The dataset comprises of check-ins from December 2010 to December 2013. This dataset was obtained through a collaboration with Foursquare.

\subsection{Formalization}

In this section, we introduce a formalization of our model. Electoral wards are the main building blocks of administrative geography in the United Kingdom; Greater London consists of 649 electoral wards and these spatial units uniquely identify London boroughs [29]. We use wards $w \in \mathbf{W}$ as a means of subdividing Greater London. We also consider venues $v \in \mathbf{V}$. A venue has a precise geographic location in a ward. A venue $v$ is represented with a tuple $v=\langle l o c, g, s\rangle$ where $l o c$ is the geographic location of the venue, $g$ its general category and $s$ is its specific category.

We define a time interval $t$ as the interval $[t \Delta,(t+1) \Delta]$ of duration $\Delta$. For example the time interval $t=0$ indicates the interval $[0, \Delta]$, the time interval $t=1$ indicates the time interval $[\Delta, 2 \Delta]$ and so on. In our work, each time interval represents distinct hours and do not overlap.

Definition 1 (Temporal profile of a ward) Similarly we define the temporal profile of a ward $w$ in an interval $[0, T]$ as the following sequence (i.e, time series):

$$
C^{w}[0, T]=\left\{c_{t}^{w}\right\} \quad \text { with } t=0,1, \ldots, T-1,
$$

where $c_{t}^{w}$ is the total number of check-ins in the ward $w$ during the time interval $t$. 
Definition 2 (Temporal profile of a venue) We define the temporal profile of a venue $v$ in an interval $[0, T]$ as the following sequence (i.e, time series):

$$
C^{v}[0, T]=\left\{c_{t}^{v}\right\} \quad \text { with } t=0,1, \ldots, T-1,
$$

where $c_{t}^{v}$ is the total number of check-ins to venue $v$ during the time interval $t$.

Definition 3 (Aggregate temporal profile of venues of a generic (specific) category in a ward) We then define $\mathbf{V}_{g, w}$ as the set of the venues of generic category $g$ in a ward $w$. Similarly, we define $\mathbf{V}_{s, w}$ as the set of the venues of specific category $s$ in a ward $w$.

Therefore, the aggregate temporal profile of venues of generic category $g$ in a ward $w$ in a time interval $[0, T]$ is defined as the following sequence (i.e, time series):

$$
C^{V_{g, w}}[0, T]=\left\{c_{t}^{g, w}\right\} \quad \text { with } t=0,1, \ldots, T-1,
$$

where $c_{t}^{g, w}$ is the total number of check-ins to venues of general category $g$ in the ward $w$ during the time interval $t$. The temporal profile of venues of a specific category in a ward can be defined in a similar way.

\section{Temporal patterns of mobile user activity}

Having formally defined the concept of temporal profiles, in this section, we discuss temporal trends of wards within Greater London and demonstrate how the composition of those wards plays a crucial role in creating a characteristic profile for that ward. We begin with an examination of all wards at one particular point in the day, highlighting that different categories dominate different wards at any given point. We then analyze more closely the characteristic temporal profile of two wards in London and discuss how their different category types contribute to their different overall profiles. We then quantify the similarity between the overall temporal profile of the 15 most popular wards in London and discuss how similarity in temporal visitation patterns could inform predictions for the temporal profile of a new venue.

\subsection{Regional temporal activity patterns}

Looking broadly at all wards within the city of London, we choose one hour of the day to highlight the idea that the popularity patterns of different neighborhoods can be dominated by different categories. Figure 1 shows the most popular category in each ward in London in the time interval $t=17$ where $\Delta=1$ (i.e., between 5 to $6 \mathrm{pm}$ ). For certain wards, this time of the day could be dominated by transport traffic as individuals commute towards home. For others, the most significant contributor could be nightlife, as individuals head to the pub for an evening drink. Similarities in the contribution of different categories to the overall temporal trend of a ward could be an indication that those wards attract individuals with similar demographics or have similar characteristics. An analysis of these similarities can be harnessed to better model, characterize, and profile different wards in a city.

Looking more closely at category types, Fig. 2 presents the characteristic temporal profile of three categories: Nightlife Spots, Colleges \& Universities, and Gyms or Fitness Centers. Each profile is a direct function of a users's propensity to visit at a given hour of the 


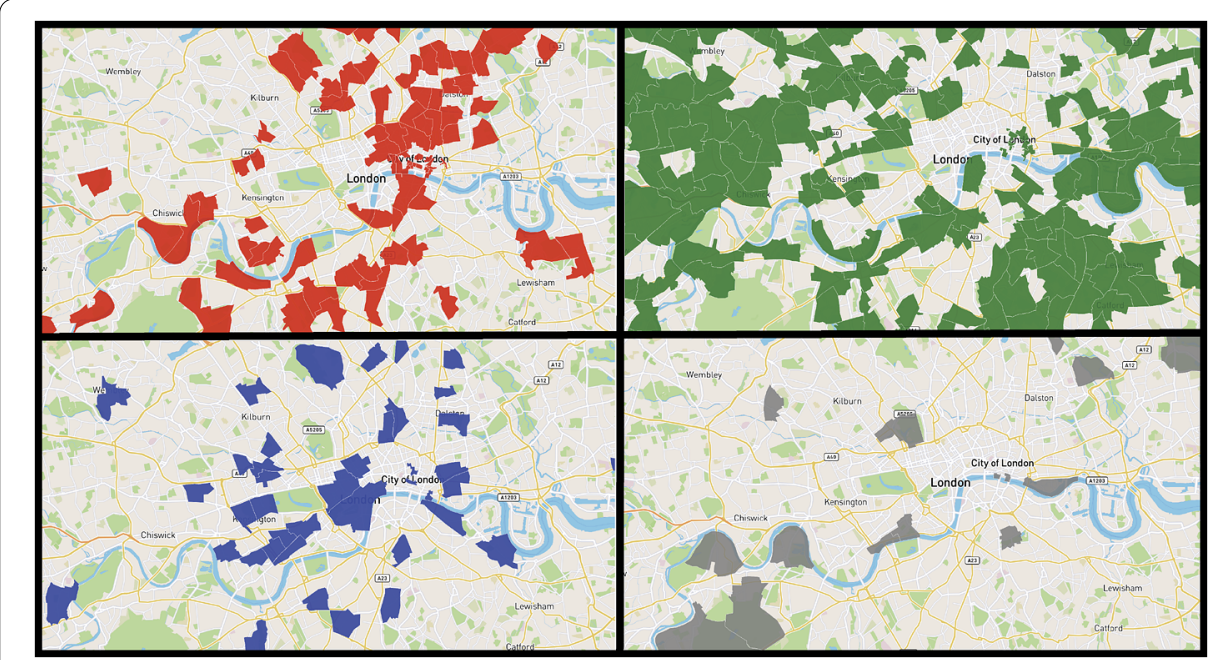

Figure 1 Venue category popularity. The most popular category in each ward at 17:00. Nightlife Spots are represented in red, Travel \& Transport in green, Food in blue, and Outdoors \& Recreation in grey

day and day of the week. The profiles of different venue categories in a ward establish the overall profile of that venue. A close examination of different wards within the city of London and of the categories which make up those wards present a number of interesting insights on how those vary in terms of their temporal visitation patterns.

In order to illustrate this let us consider two wards of interest: St. Pancras \& Somers Town, which contains a major transportation hub, offices, and academic institutions, and Camden Town with Primrose Hill, which contains a variety of venues and tourist attractions. Figure 3 shows the average number of check-ins in each ward for each hour of the day over the course of one week, aggregating across a number of weeks. This signal creates a characteristic temporal profile which acts as a temporal signature for the ward. The overall signal, shown in black, is different for these two wards. The number of check-ins at Camden Town steadily increases over the course of the day while the number check-ins at St. Pancras has two large peaks, one in the morning and another in the evening. Examining the three main categories (Food, Travel \& Transport, and Nightlife Spots) that characterize these two wards can help to better understand this observation. Camden Town has significant contributions from Nightlife venues which gradually increases over the course of a day. Conversely, St. Pancras is dominated by Travel \& Transport, causing the overall temporal profile of the ward to peak at rush hour. These trends suggest that Camden Town is likely a more youth dominated area while St. Pancras is a hub for commuters or travelers, as they actually are [30].

\subsection{Utilizing similarities in visitation patterns}

Similar observations can be generalized to the rest of the wards in London. Different regions feature different degrees of similarity, an insight which we exploit in Sect. 6 to predict the characteristic temporal curves of new venues. We quantify the similarity between two temporal profiles using the Jensen-Shannon divergence (JSD) [31]. We use the JSD instead of the Kullback-Leibler divergence (KLD) since the former is a symmetric similarity measure between two functions whereas the latter is not. Our analysis showed that the JSD improved our percent accuracy by 7 to $10 \%$ over the KLD. The JSD between two wards $w_{i}$ 


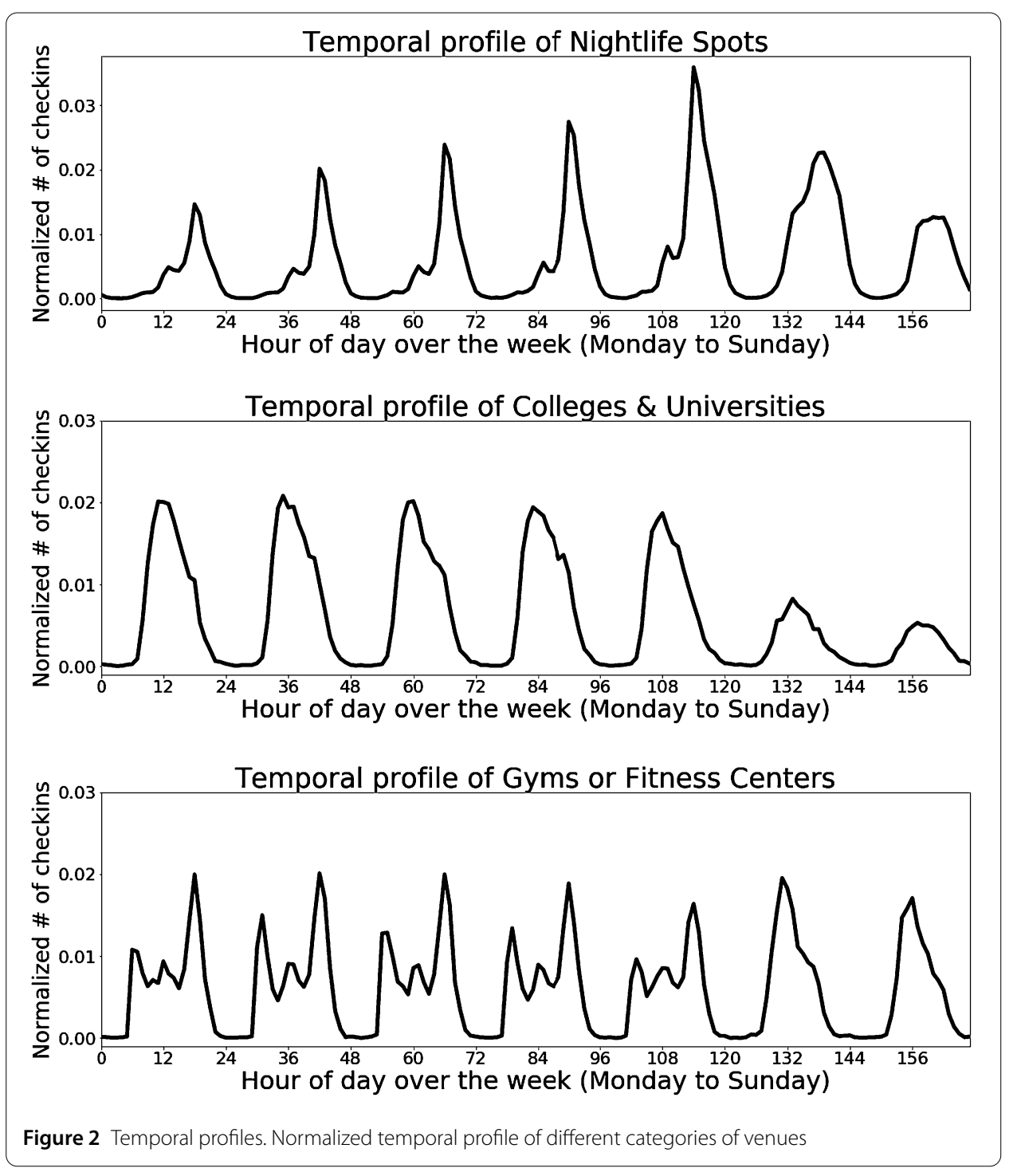

and $w_{j}$ is calculated as follows:

$$
\operatorname{JSD}\left(C^{w_{i}}, C^{w_{j}}\right)=H\left(\frac{C^{w_{i}}+C^{w_{j}}}{2}\right)-\frac{H\left(C^{w_{i}}\right)+H\left(C^{w_{j}}\right)}{2},
$$

where $H$ is the Shannon entropy. The JSD provides an information-theoretic metric that quantifies how two profiles, which that can be seen as distributions over time, are similar. A low value of the JSD between the temporal profile of two wards represents a high similarity.

In Fig. 4 we present the Jensen-Shannon divergence between the temporal profiles of the 15 most popular wards in London with regards to their total number of check-ins. There is an evident range in similarity between the various wards. For instance, Hyde Park is very similar to St. Pancras and Somers Town as both are central travel hubs that handle the large commuter flows to local corporate offices and government buildings. Other characteristic examples are wards such as St. James's and West End that attract a large tourist population visiting the attractions in the respective areas. These results suggest that similarities 


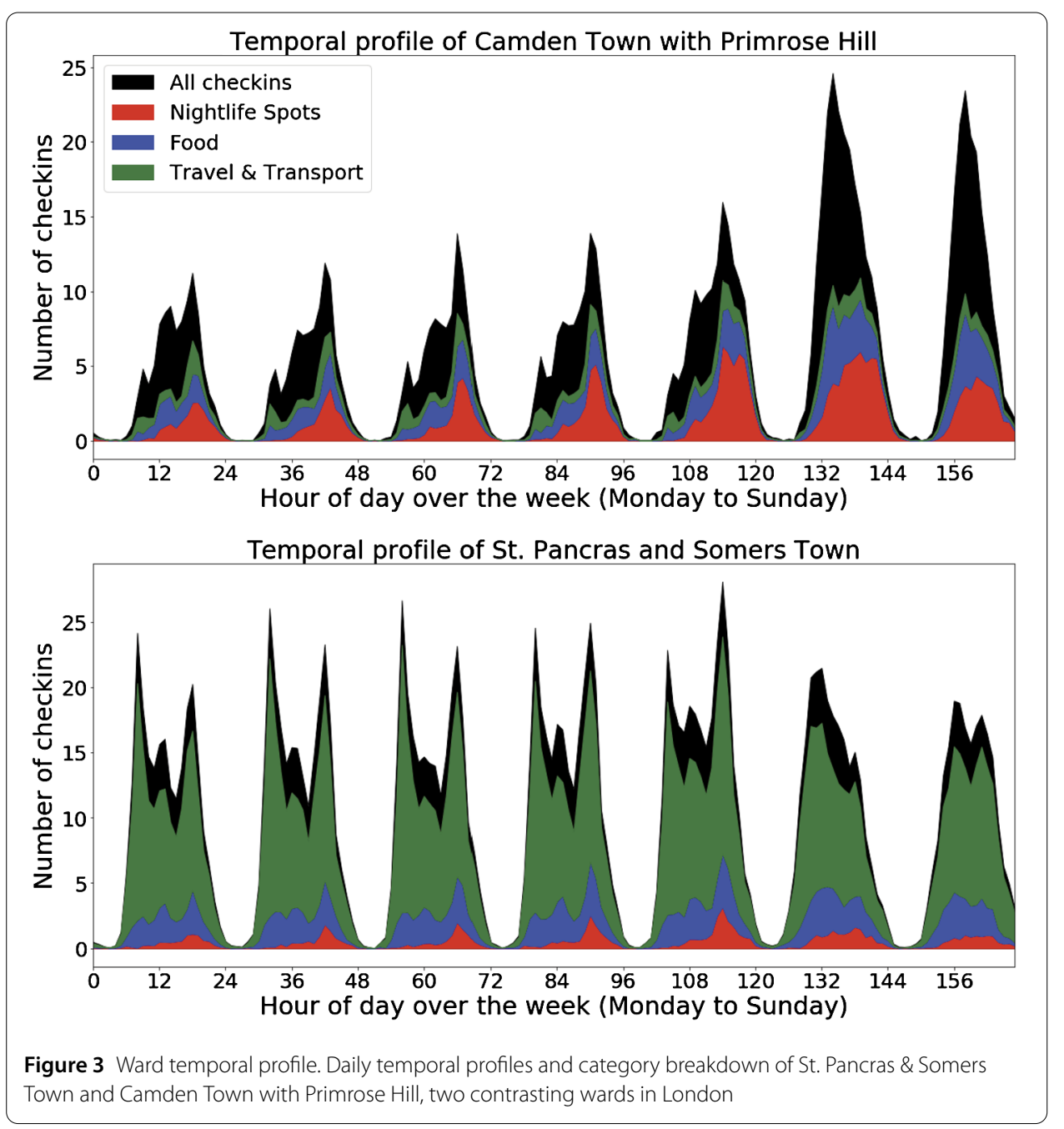

in temporal profiles can be useful indicators of similarities in the characteristics of two wards. We aim to use this insight for our overarching goal of predicting the characteristic temporal profile of a new venue. This similarity can be used for prediction.

First, we explore how the temporal profile of a new venue becomes more and more stationary with each consecutive week and then demonstrate how similar wards can be used for the prediction task.

\subsection{Temporal visitation patterns of new venues}

We now focus on the temporal characteristics of new venues. These venues represent an interesting case study as upon their launch, unlike existing venues or geographic areas, there is no historic information on their expected popularity patterns over time. We introduce next basic properties of the new venues data that we use during evaluation. We demonstrate how their temporal profiles converges to a stationary state over time, a process that will let us define the prediction task presented in Sect. 6 .

Identification of new venues. The Foursquare dataset includes a list of all public venues in the city of London. Critically for the present work the creation time of every venue is available in the database. The creation time refers to the date the venue was crowdsourced by Foursquare users. Prior research on Foursquare data has shown that venues added after 


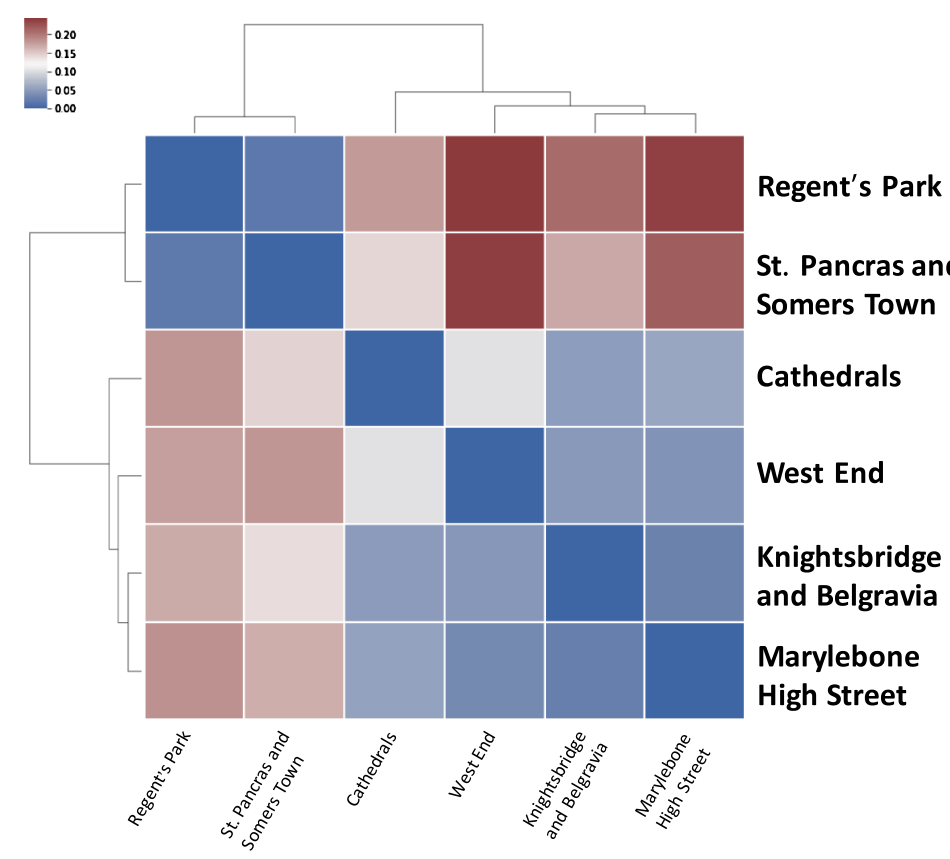

Figure 4 Temporal similarity of wards. J-S divergence of the characteristic weekly temporal profile of the 15 most popular wards. Smaller values signify a smaller divergence and thus more similarity

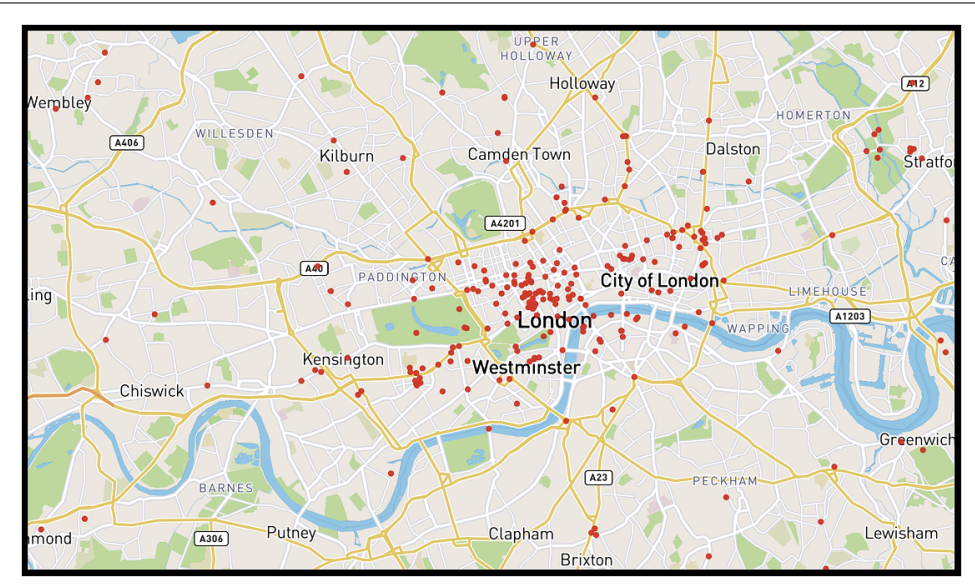

Figure 5 Set of new venues. Coordinates of the set of 305 new venues in London considered in the study

June 2011 were highly likely (probability above 0.8 ) to actually be new venues opening in an area rather than existing venues being added to the system for the first time [17]. We look at all new venues that were added to Foursquare after June 2011 that had at least 100 check-ins. This results in a list of 305 venues which is used for the following analysis. Within this list of newly opened venues, $32 \%$ of the venues fall within the general category of Food, 32\% under Travel E Transport, and 8\% under Nightlife Spots. These venues were scattered around the city, with their focal point being in center of the city as depicted in Fig. 5.

Defining a venue's stationary temporal profile. For each of the new venues in our set, we first examine the total number of checkins at each time step for each week after the venue 
opened (i.e. the weekly temporal profile). To avoid sparsity issues working at this level of granularity, we create a cumulative temporal profile per week, summing the total number of checkins at each time step with each consecutive week. Although the raw values of cumulative sums have little significance, the trend over the course of the week represents the characteristic curve of the venue and indicates the weekly demand trend. We normalize the curve for each week by dividing by the sum of all checkins for the venue, up to the time of observation. With each consecutive week, we expect this curve to show a higher degree of stationarity. We measure the stationarity of this temporal profile over time by calculating the variance of the temporal curve at time $t$ relative to time $t-1$.

Our data suggests that the temporal profile of a new venue becomes stationary when the value of the variance relative to the prior week is $\sigma^{2}<2.6 \times 10^{-5}$. On average, this occurs 5 weeks after a venue has opened. Note that we build the profile of a venue considering a week's temporal span. This captures the most essential temporal patterns of activity at a venue, which includes diurnal variations, but also differences between weekends and weekdays.

\section{Predicting the temporal signature of new venues}

Having built an understanding of similarities in the temporal profile of categories and wards, we aim to apply these findings to predict the stationary temporal profile of a new venue. We adopt a $k$-nearest neighbors approach in which we find the $k$ most temporally similar wards to the ward in which the new venue is located. We look not only at the overall profile of wards as a means of comparison but also at the profile of categories within those wards. The temporal profile of those wards serve as predictors for the new venue and are used to train a Gaussian Process model [32].

\subsection{Discovering area-wide similarities in popularity dynamics}

We have seen that similarities in the temporal profiles of wards can be useful indicators of similarities in the characteristics of two wards (Sect. 5.2). We use this idea for our model in which we begin with the basis that two venues of the same category in two different wards are likely to have similar temporal patterns if the overall temporal patterns of their wards are similar. This idea is illustrated in Fig. 6 which shows the stable temporal profile of a new venue $v_{i}$ and the temporal profile of the five most similar wards. Further, Fig. 7 shows the normalized root mean squared error (NRMSE) between the stable profile of a new venue and each of the five similar wards as well as between the profile output from the GP. The figure demonstrates that GP predictors provides a better prediction with respect to simply using the profiles of similar wards.

For a given new venue $v_{i}$, our methodology to predict its temporal profile is as follows. For clarity, we will describe an example in which we assume $v_{i}$ is an Italian restaurant called The Meaning of Life in ward 42.

1. Determine the general category, specific category, and ward of that venue. For our example, the general category is Food, the specific category is Italian restaurant, and the ward is 42 .

2. Determine the temporal profile of the ward for the general category of interest. In this example, we would determine the overall temporal profile of Food venues in ward 42. Formally, we determine $C^{V_{g, w}}[0, T]$ where $T=168$. 

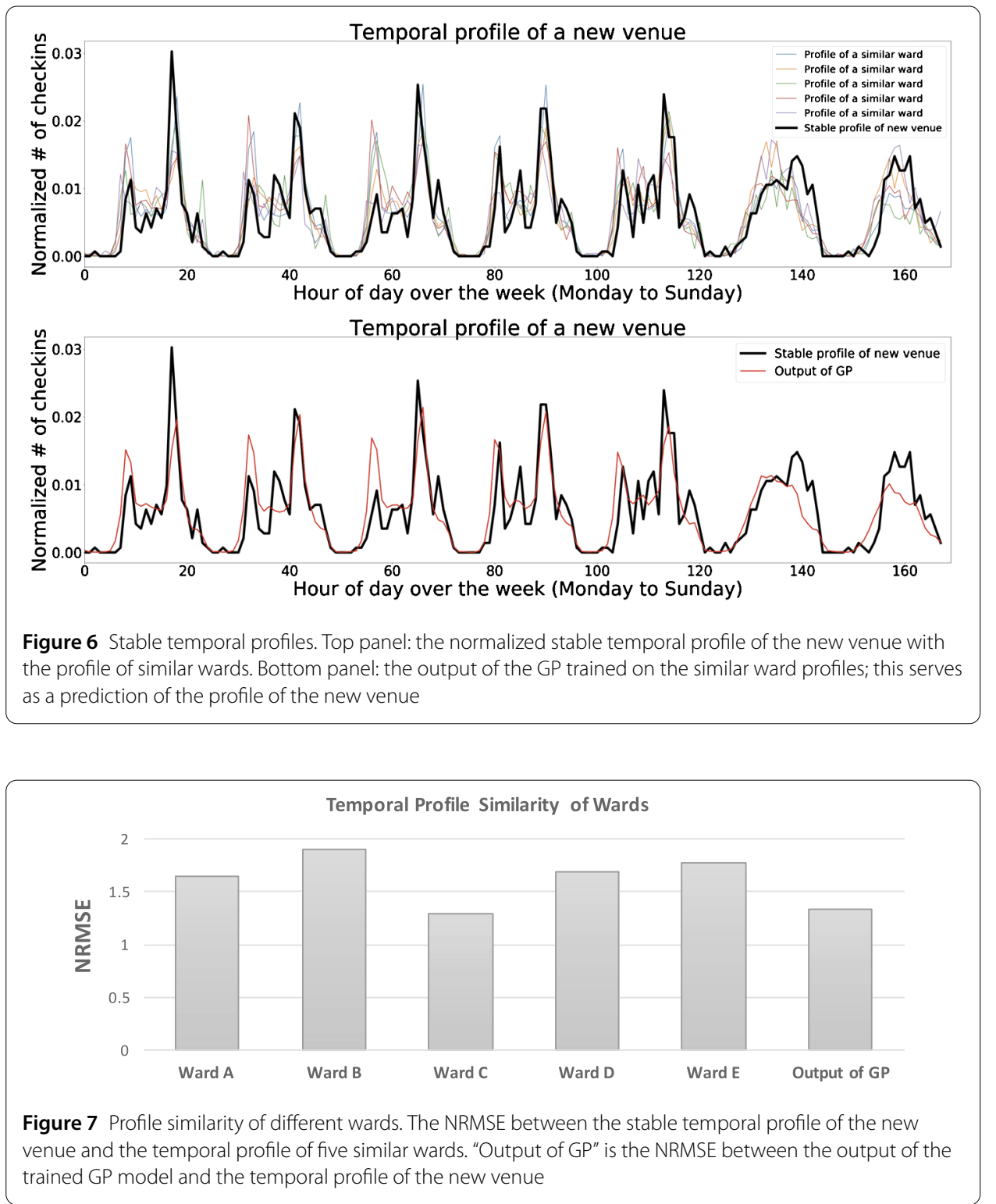

3. Determine the $N$ most similar wards. For all other wards in the city, compare their general category's temporal profile to that of our ward of interest and determine the $N$ most similar wards where similarity is defined as $\operatorname{JSD}\left(C^{v}, C^{w}\right), v \neq w$. This is referred to as the set of temporally similar wards. For our example, this would entail finding the $N$ wards whose Food temporal profile is most similar to that of ward 42 .

4. Calculate the specific temporal profile for each ward in the set of temporally similar wards. For our example, this would mean we would calculate the temporal profile of Italian restaurants for each of the $N$ similar wards.

5. Create a representative curve. These $N$ temporal curves serve as the basis of our prediction of the profile of our new venue $v_{i}$. To create a representative curve from those $N$ profiles, we use each of the profiles as inputs to a Gaussian Process (GP) because of its ability to recognize latent periodic trends. The output from the GP becomes our temporal prediction. 


\subsection{Gaussian processes model}

Our $k$-nearest temporal neighbors algorithm finds temporal profiles that are likely to be similar to the venue of interest. We harness Gaussian Processes to build a regression model to capture the periodic trends in those profiles. GPs are typically employed in time-series tasks because of their flexibility at capturing the complex structures without succumbing to overfitting. GP regression is a Bayesian non-parametric which models a distribution over an infinite set of random variables. A GP model is described by its prior mean and covariance functions. For this work, as is standard, we set the prior mean to zero [32]. For the analysis of this we use a product of two Radial Basis function kernels as the base kernel functions which define the covariance matrix of the distribution. We use two kernels to describe two types of periodicity in our data, over the course of a week as well as over the course of a day.

Given the periodicity over the course of a day and a week, we posit that Gaussian Processes are able to recognize latent periodic trends in the data. The inputs to our Gaussian Process are the temporal profiles of the similar wards. We then have the GP predict a temporal pattern for an interval of $[0, T]$ where $T=168$ for the hourly week's profile. We then compare this prediction to stable temporal profile of the venue of interest.

\subsection{Evaluation}

In this section, in this section we present an evaluation of our algorithm introducing first a set of baselines as comparators and metrics for comparing the experimental results.

Baselines. To evaluate our proposed solution, we compare our results with a number of baseline approaches. For each, the past temporal profiles (i.e., earlier than the venue creation time) are used as features for a GP and the outputs of the GP are the prediction of the characteristic temporal profile of the new venue. The baselines and their descriptions are listed in Table 1.

Metrics. To analyze the accuracy of our prediction, we calculate the NRMSE between the predicted temporal profile and the stable profile for each venue. We first look at the value of NRMSE as we vary the number of neighbors $N$. Our results show $N=10$ to be the best indicator of temporal similarity of neighbors. This value was chosen for the subsequent analysis presented in this paper.

Results. Using $N=10$, we calculate the normalized root mean squared error for the output of each algorithm compared to the actual stable curve of each new venue. Table 1 presents a summary of these results. Temporally similar wards using the specific category of the venue proves to be the best predictor of the temporal profile of a new venue.

Table 1 Comparative analysis of different similarity criteria

\begin{tabular}{lll}
\hline Criteria & Description of Criteria & NRMSE \\
\hline TempGen & Temporally similar wards, same general category & 1.614 \\
TempSpec & Temporally similar wards, same specific category & $\mathbf{1 . 5 7 5}$ \\
Random & Random wards & 2.692 \\
SameAl1 & Same ward, all categories & 2.1941 \\
SameGen & Same ward, same general category & 1.884 \\
SameSpec & Same ward, same specific category & 1.760 \\
AllAll & All wards, all categories & 1.937 \\
AllGen & All wards, same general category & 2.190 \\
AllSpec & All wards, same specific category & 2.028 \\
\hline
\end{tabular}




\section{On-line prediction of mobility trends at new venues}

As demonstrated in the previous section, predicting the stable temporal curve of a venue is possible and can become more accurate when temporal information from other venues or areas is selectively transferred. Human mobility patterns, despite being characterized by a high degree of regularity, can change over time. Although the characteristic temporal curve provides an indication of the typical temporal trends, the ability to predict demand in real-time can directly help a shop owner to dynamically approximate demand trends, even during the first few months after a venue has opened. In this section, we aim to forecast the popularity of a venue and predict the success of that venue after it has opened. On a day to day basis, we expect the profile of check-ins to vary, however, the variation in demand on a per month basis is less susceptible to noise and a more reliable indicator of the success of a new venue.

\subsection{On-line prediction task}

When working at the granularity of individual venues in an on-line manner, the sparsity of the data becomes a real concern. In order to address this issue, for each venue, we aggregate check-ins over the course of a month. Predicting a significant increase or decrease in demand for a venue for the following month is useful information for a shop owner to know as it can inform crucial business decisions and who are unlikely to have historic data for the venue. To inform our predictions we utilize locality, the past demand trends of venues in our neighborhood, and temporal similarity, the demand trends of temporally synchronous areas of the city. We next provide a roadmap of how local trends in user mobility can be exploited to forecast alterations of traffic at individual venues in future months.

For each new venue, our prediction task is to estimate the change in demand for each month following the first month of business; then, starting at the first week in which it opened, we aim to predict whether the demand of the venue at its next time step will increase, decrease, or remain stable (i.e., a 3-class prediction task). Remaining stable is defined as remaining within ten percent of the prior value. It is worth noting that the proposed methodology does not depend on the choice of this interval. With each subsequent month since a venue has opened, we have more data with which we can better understand the venue.

We inform our predictions by selectively using temporal information from other areas of the city. A number of different criteria were used when selecting these inputs, which are listed in Table 2. For example, when using the "history" of a venue, for each month after the

Table 2 AUC values of the real-time prediction with a varying number of months of training data

\begin{tabular}{llllll}
\hline & 2 & 3 & 4 & 5 & 6 \\
\hline History & 0.6748 & 0.6697 & 0.6853 & 0.7286 & 0.7278 \\
TempGen & 0.7507 & 0.7820 & 0.7691 & 0.7824 & 0.7903 \\
TempSpec & 0.7729 & 0.7804 & 0.7829 & 0.7991 & 0.8104 \\
Random & 0.5102 & 0.5185 & 0.5248 & 0.5682 & 0.5993 \\
SameAl1 & 0.7149 & 0.7310 & 0.7382 & 0.7349 & 0.7352 \\
SameGen & 0.7403 & 0.7481 & 0.7592 & 0.7480 & 0.7791 \\
SameSpec & $\mathbf{0 . 7 8 5 9}$ & $\mathbf{0 . 7 9 1 5}$ & $\mathbf{0 . 7 9 8 1}$ & $\mathbf{0 . 8 2 1 6}$ & $\mathbf{0 . 8 2 2 1}$ \\
AllAl1 & 0.6812 & 0.6892 & 0.6489 & 0.6893 & 0.6832 \\
AllGen & 0.6824 & 0.6853 & 0.6935 & 0.7088 & 0.7129 \\
AllSpec & 0.7201 & 0.7209 & 0.7403 & 0.7459 & 0.7402 \\
\hline
\end{tabular}


venue opens, we train a GP on the demand for each month. We then predict the relative demand at each progressive month, continuously learning from the previous months (i.e., month 1 to 3 would be used in the training dataset when predicting month 4). This realtime prediction methodology harnesses the Bayesian nature of GPs and their ability to predict and react to anomalies in the data (i.e., if there is a sharp peak at a given time step $t$ the GP uses that insight when predicting $t+1$ ). This mirrors situations in which the demand of a venue can sharply increase because of events on a given day.

\subsection{Evaluation of the on-line prediction task}

We predict the relative demand curve in real-time for one week for each of our 305 new venues. Table 2 presents the AUC value when predicting the relative demand curve after the training phase.

Our baseline is to train the model on the history of the new venue after the first month from it's opening. We examine the use of temporally similar wards as a predictor. We use as our prediction the history of 10 wards because this value provided the optimal representation of similarity. We also examined as inputs venues in wards that have the same general and specific category as the venue of interest. Our results show that locality does have an impact on predictions as the predictions using venues in the same ward are higher than using venues in all wards throughout the city. Further, we see that temporal similarity can also be used to improve predictions; this may be because similar venues could have synchronous peaks in demand following a similar impact from real-world events.

\section{Discussion}

The evaluation results discussed in the previous section have shed new light on the temporal dynamics of user activity in location-based services.

At neighborhood level, we have seen that areas that are far from each other can be synchronized with regards to their temporal activities. Moreover, the temporal frequencies of such activities tend to be stationary over certain periods of time due to regularities in human mobility patterns. We exploited this information to predict the temporal popularity profiles of newly established venues in Sect. 6, essentially transferring information from the level of an urban region to that of a specific venue. This form of analytics can provide new insights to new business owners who can plan supplies and staffing in their facilities during the cold start period of a new opening. Beyond retail venues, the idea can be expanded to other types of places, such as parks or outdoor spaces. Predicting how urban spaces are used over time can improve planning, including the design of schedules for their maintenance or police them. Despite the regularity patterns observed in human mobility, variations over time will exist due to social events or unforeseen circumstances such as travel disruptions. These can result in historically unexpected increases or decreases in mobility flows towards a venue.

To examine whether such variations can be captured on a venue level we experimented with an on-line prediction task, where the goal was to predict relative changes with respect to historic patterns of a venue. This is a challenging task from the point of view of data sparsity. Even very popular venues in location-based services will have only a handful of check-ins observed in a small time window. We have demonstrated that it is possible to pick up trends in this setting using a Gaussian Process model trained on data inputs from recent mobile user activity at nearby venues. 


\section{Conclusion and future work}

In this work, we have investigated the prediction of the temporal dynamics of newly established venues using the check-in data of millions of Foursquare users. We have also introduced the concept of temporally similar areas in a city, areas that share patterns in the movement of people to different types of venues within those areas.

We have shown that the characteristic temporal curve of a new venue provides valuable insight for new shop owners who can use that information to better inform supply purchases, opening hours, and demand. Characteristic curves can also support the design of location-based technologies. Additionally, our models help to demonstrate how a particular venue influences the overall temporal profile of the neighborhood it is located in. This knowledge can help design more interpretable models and build urban applications that are aware of the behavioral choices made by citizens on a local level, rather than those that treat population dynamics as a blind optimization task.

We next plan to study venues with different demand characteristics-not only the most popular venues. Less popular venues may attract different demographics or present unconventional temporal properties. Additionally, future work includes the analysis of cities around the world in order to understand their regularity and temporal trends through the application of the methodology discussed in this paper. The framework proposed in this paper can be applied to different cities, since it is not based on any assumptions regarding the spatial and urban context. Indeed, in order to apply the framework, a different model has to be trained, potentially with different values of $k$ and the hyperparameters of the Gaussian Process. However, in order to select these values, it would be sufficient to follow the methodology described in this paper.

Acknowledgements

We thank Foursquare for supporting this research by providing the dataset employed in the analysis.

Funding

This work was supported through the Gates Cambridge Trust and partially supported by the Alan Turing Institute under the EPSRC grant EP/N510129/1. The work was also supported by the EPSRC through Grant GALE (EP/K019392).

Abbreviations

LBSNs, Online location-based social networks; JSD, Jensen-Shannon divergence; GP, Gaussian process; NRMSE, Normalized root mean squared error.

Availability of data and materials

The Foursquare dataset used in this work has been shared by the company through an official agreement with the University of Cambridge that we have no authority to redistribute it.

Competing interests

The authors declare that they have no competing interests.

Authors' contributions

All authors discussed and designed the experiments as well as contributing to the write up of the paper. KD carried out the computational tasks, analyzed the data and prepared the figures. All authors read and approved the final manuscript.

\section{Author details}

${ }^{1}$ Department of Computer Science, University of Cambridge, Cambridge, UK. ${ }^{2}$ Center for Data Science, New York University, New York, USA. ${ }^{3}$ Department of Geography, University College London, London, UK. ${ }^{4}$ The Alan Turing Institute, London, UK. ${ }^{5}$ Foursquare Labs, New York, USA.

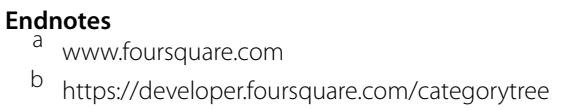

b https://developer.foursquare.com/categorytree

\section{Publisher's Note}

Springer Nature remains neutral with regard to jurisdictional claims in published maps and institutional affiliations. 
Received: 22 January 2018 Accepted: 10 May 2018 Published online: 18 May 2018

\section{References}

1. Kaiser EJ, Godschalk DR, Chapin FS (1995) Urban land use planning. University of Illinois Press, Urbana

2. Batty M (2007) Cities and complexity: understanding cities with cellular automata, agent-based models, and fractals. MIT Press, Cambridge

3. Reades J, Calabrese F, Sevtsuk A, Ratti C (2007) Cellular census: explorations in urban data collection. IEEE Pervasive Comput 6(3):30-38

4. Calabrese F, Colonna M, Lovisolo P, Parata D, Ratti C (2011) Real-time urban monitoring using cell phones: a case study in Rome. IEEE Trans Intell Transp Syst 12(1):141-151

5. Ratti C, Frenchman D, Pulselli RM, Williams S (2006) Mobile landscapes: using location data from cell phones for urban analysis. Environ Plan B, Plan Des 33(5):727-748

6. Becker R, Cáceres R, Hanson K, Isaacman S, Loh JM, Martonosi M, Rowland J, Urbanek S, Varshavsky A, Volinsky C (2013) Human mobility characterization from cellular network data. Commun ACM 56(1):74-82

7. Jiang S, Yang Y, Gupta S, Veneziano D, Athavale S, González MC (2016) The TimeGeo modeling framework for urban motility without travel surveys. Proc Natl Acad Sci USA 113(37):E5370-E5378

8. França U, Sayama H, McSwiggen C, Daneshvar R, Bar-Yam Y (2016) Visualizing the "heartbeat" of a city with tweets. Complexity 21(6):280-287

9. Silva TH, Vaz de Melo PO, Almeida JM, Salles J, Loureiro AA (2013) A comparison of Foursquare and Instagram to the study of city dynamics and urban social behavior. In: Proceedings of the 2nd ACM SIGKDD international workshop on urban computing. ACM, New York, p 4

10. Zheng Y, Capra L, Wolfson O, Yang H (2014) Urban computing: concepts, methodologies, and applications. ACM Trans Intell Syst Technol 5(3):38

11. Singh VK, Bozkaya B, Pentland A (2015) Money walks: implicit mobility behavior and financial well-being. PLoS ONE 10(8):e0136628. https://doi.org/10.1371/journal.pone.0136628

12. Jensen $P$ (2006) Network-based predictions of retail store commercial categories and optimal locations. Phys Rev $E$ 74(3):035101

13. Karamshuk D, Noulas A, Scellato S, Nicosia V, Mascolo C (2013) Geo-spotting: mining online location-based services for optimal retail store placement. In: Proceedings of the 19th ACM SIGKDD international conference on knowledge discovery and data mining

14. Shaw B, Shea J, Sinha S, Hogue A (2013) Learning to rank for spatiotemporal search. In: Proceedings of the 6th ACM international conference on web search and data mining (WSDM '13). ACM, New York, pp 717-726

15. Noulas A, Scellato S, Mascolo C, Pontil M (2011) An empirical study of geographic user activity patterns in Foursquare. In: Proceedings of the fifth international AAAI conference on weblogs and social media (ICWSM '11)

16. Pan B, Zheng Y, Wilkie D, Shahabi C (2013) Crowd sensing of traffic anomalies based on human mobility and social media. In: Proceedings of the 21st ACM SIGSPATIAL international conference on advances in geographic information systems (SIGSPATIAL '13). ACM, New York, pp 344-353

17. Daggitt M, Noulas A, Shaw B, Mascolo C (2016) Tracking urban activity growth globally with big location data. R Soc Open Sci 3(4):150688

18. Silva TH, de Melo PO, Almeida J, Musolesi M, Loureiro A (2014) You are what you eat (and drink): identifying cultural boundaries by analyzing food \& drink habits in Foursquare. In: Proceedings of the 8th international AAAI conference on weblogs and social media (ICWSM '14)

19. Google (2017) Popular times and visit duration. https://support.google.com/business/answer/6263531

20. Facebook (2017) Discover great places in every city. https://www.facebook.com/places/

21. Fu Y, Ge Y, Zheng Y, Yao Z, Liu Y, Xiong H, Yuan J (2014) Sparse real estate ranking with online user reviews and offline moving behaviors. In: Proceedings of the 2014 IEEE international conference on data mining (ICDM '14). IEEE, New York, pp 120-129

22. Roth C, Kang SM, Batty M, Barthélemy M (2011) Structure of urban movements: polycentric activity and entangled hierarchical flows. PLoS ONE 6(1):15923

23. Smith C, Quercia D, Capra L (2013) Finger on the pulse: identifying deprivation using transit flow analysis. In: Proceedings of the 2013 conference on computer supported cooperative work (CSCW '13). ACM, New York, pp 683-692

24. Balan RK, Nguyen KX, Jiang L (2011) Real-time trip information service for a large taxi fleet. In: Proceedings of the 9th international conference on mobile systems, applications, and services (MobiSys '11). ACM, New York, pp 99-112

25. Santi P, Resta G, Szell M, Sobolevsky S, Strogatz SH, Ratti C (2014) Quantifying the benefits of vehicle pooling with shareability networks. Proc Natl Acad Sci USA 111(37):13290-13294

26. Rajagopalan HK, Saydam C, Xiao J (2008) A multiperiod set covering location model for dynamic redeployment of ambulances. Comput Oper Res 35(3):814-826

27. Sheu J-B (2007) An emergency logistics distribution approach for quick response to urgent relief demand in disasters. Transp Res, Part E, Logist Transp Rev 43(6):687-709

28. VentureBeat (2015) Foursquare by the numbers. https://goo.gl/Vi1UUf

29. Office for National Statistics (2011) Number of electoral wards/divisions in the United Kingdom. https://www.ons.gov.uk/

30. Greater London Authority (2014) LSOA Atlas. https://data.Iondon.gov.uk/dataset/lsoa-atlas

31. Lin J (1991) Divergence measures based on the Shannon entropy. IEEE Trans Inf Theory 37(1):145-151

32. Rasmussen CE, Williams C (2006) Gaussian processes for machine learning. MIT Press, Cambridge 